\title{
Preparation of heteromacrocycles bearing two tetra-coordinate phosphorus atoms and at least two ferrocene units
}

\author{
Alberto Tárraga*, Pedro Molina*, and David Curiel \\ Departamento de Química Orgánica, Facultad de Química, Universidad de Murcia, \\ Campus de Espinardo, E-30071 Murcia, Spain \\ E-mail:pmolina@um.es
}

\section{Dedicated to Prof. M. Moreno Mañas on occasion of his $60^{\text {th }}$ birthday (received 20 Dec 01; accepted 20 Feb 02; published on the web 28 Feb 02)}

\begin{abstract}
A synthetic procedure has been developed for the preparation of a new type of macrocycle in which the ferrocene unit is appended to an O-N-P-macrocycle or it is incorporated within the macrocyclic unit. The methodology is based on the Staudinger reaction of a bis-azide with the appropriate diphosphine.
\end{abstract}

Keywords: Heteromacrocycles, ferrocene, Staudinger reaction, bis-azide, diphosphine

\section{Introduction}

Recent years have witnessed a significant interest in a new generation of abiotic macrocycles and cryptans that contain an electrochemical response function, such as the ferrocene unit, either attached to, or as integral part of, the ligand framework. By suitable variation of the size and structure of the host cavity, these molecules can be made to bind several kind of ionic or neutral guest species giving rise to supramolecular systems that have potential applications in several fields. ${ }^{1}$ In particular, these ligands are of special interest in the construction of heterobimetallic or complexing host systems, which can behave as redox-active and photo-active receptors with the capability of selectively sensing ionic or neutral guest species via electrochemical and/or optical methodologies. ${ }^{2}$ In this context, by introducing the iminophosphorane moiety as part of the macrocyclic ring, improvements can be made, since it is known its high complex-forming capability with a wide variety of metal compounds ${ }^{3}$ and metal carbonyls. ${ }^{4}$

We recently reported a new approach to the preparation of a new type of macrocycles bearing a disubstituted ferrocene moiety ${ }^{5}$ and a kind of $P, N$-heterodifunctional ferrocene-coordinating open chain ligands ${ }^{6}$ that is based on the Staudinger imination ${ }^{7}$ of trivalent phosphorus compounds with azides to produce an iminophosphorane function after nitrogen evolution. 
Although the synthesis of phosphorus-containing macrocycles possessing an intracyclic C-P-N grouping have been previously reported ${ }^{8}$ we wish to report the preparation of macrocycles containing two tetra-coordinate phosphorus atoms bearing two iminophosphorane moieties [macrocyclic bis(iminophosphoranes)] as well as the ferrocene unit both appended to the macrocycle or incorporated within the macrocycle framework. The methodology used is based on the reaction of the bis-azide 3 with diphosphines of general structure $\mathrm{R}_{2} \mathrm{P}-\mathrm{Y}-\mathrm{PR}_{2}$ some of which have been used by Majoral and coworkers for the preparation of phosphorus-containing cryptands. ${ }^{9}$ Frequently, the synthesis of macrocycles with chemically diverse donating (or bonding) atoms requires multistep manipulations with concomitantly low yields or difficult separations. However the macrocyclization process following this methodology takes place in a single step, with high yields and without the presence of any template. Additional advantages of this process would be provided by the possibility of tuning the size of the cavity by systematic modification both the $\alpha, \omega$-diaminoderivatives used in the preparation of the starting material and the diphosphine spacer, and by the possibility of preparing ferrocene-derived phosphines in which the phosphorus atom is not directly bonded to a cyclopentadienyl ring, which is not a very common situation. $^{10}$

\section{Results and Discussion}

During the course of a recent project aimed at the preparation of ferrocenyl-substituted azaheterocycles we reported the preparation of ethyl 2-azido-3-ferrocenylacrylate 1, by condensation of ferrocenecarboxaldehyde and ethyl azidoacetate. ${ }^{11}$ Hydrolysis of the ester group followed by treatment of the resulting carboxylic acid 2 with oxalyl chloride, in dichloromethane at room temperature, gives rise to the expected 2-azido-3-ferrocenylacroyl chloride, pure enough to be used in the next step without further purification. Reaction of the acyl chloride with 2,2'(ethylenedioxy)bis(ethylamine) in dry THF and in the presence of triethylamine afforded the corresponding bis-azidoacrylamide 3 in 55\% yield, which in turn was reacted with the appropriate diphosphine to give the macrocyclic derivatives 4a-c. To the best of our knowledge, these are the first examples of compounds in which two iminophosphorane linkages are present in the macrocyclic framework together with two or three ferrocene units, respectively.

It is worth noting the high yields obtained for the [1+1] macrocyclization process, which could be explained by an endo-template effect, taking into account that an internal hydrogen bond could be formed between the amide $\mathrm{NH}$ group and the oxygen atoms present in the bridge $\mathrm{e}^{12}$ or between such NH group and the nitrogen present in the iminophosphorane moiety. ${ }^{13}$

The ${ }^{1} \mathrm{H}-\mathrm{NMR}$ spectra of compounds $\mathbf{4 a}$ and $\mathbf{4 b}$ show characteristic features: the ferrocenyl hydrogen atoms give a singlet at $\delta=4.00-4.06$ for the unsubstittued cyclopentadienyl ring and two singlets at $\delta=3.95-4.04$ and $\delta=4.43-4.58$, corrresponding the monosubstituted ring, while the vinylic proton signal appears as a singlet at $\delta=6.18-6.22$. The ${ }^{13} \mathrm{C}-\mathrm{NMR}$ spectra also exhibit the signals for monosubstituted ferrocenes, and the FAB mass spectra gives the expected $\left[\mathrm{M}^{+}+1\right]$ 
peaks at $\mathrm{m} / \mathrm{z}=1049$ and 1065 , respectively. The ${ }^{31} \mathrm{P}-\mathrm{NMR}$ spectra of $\mathbf{4 a}$ and $\mathbf{4 b}$ exhibit one singlet at $\delta=4.97$ and $1.17 \mathrm{ppm}$, respectively.
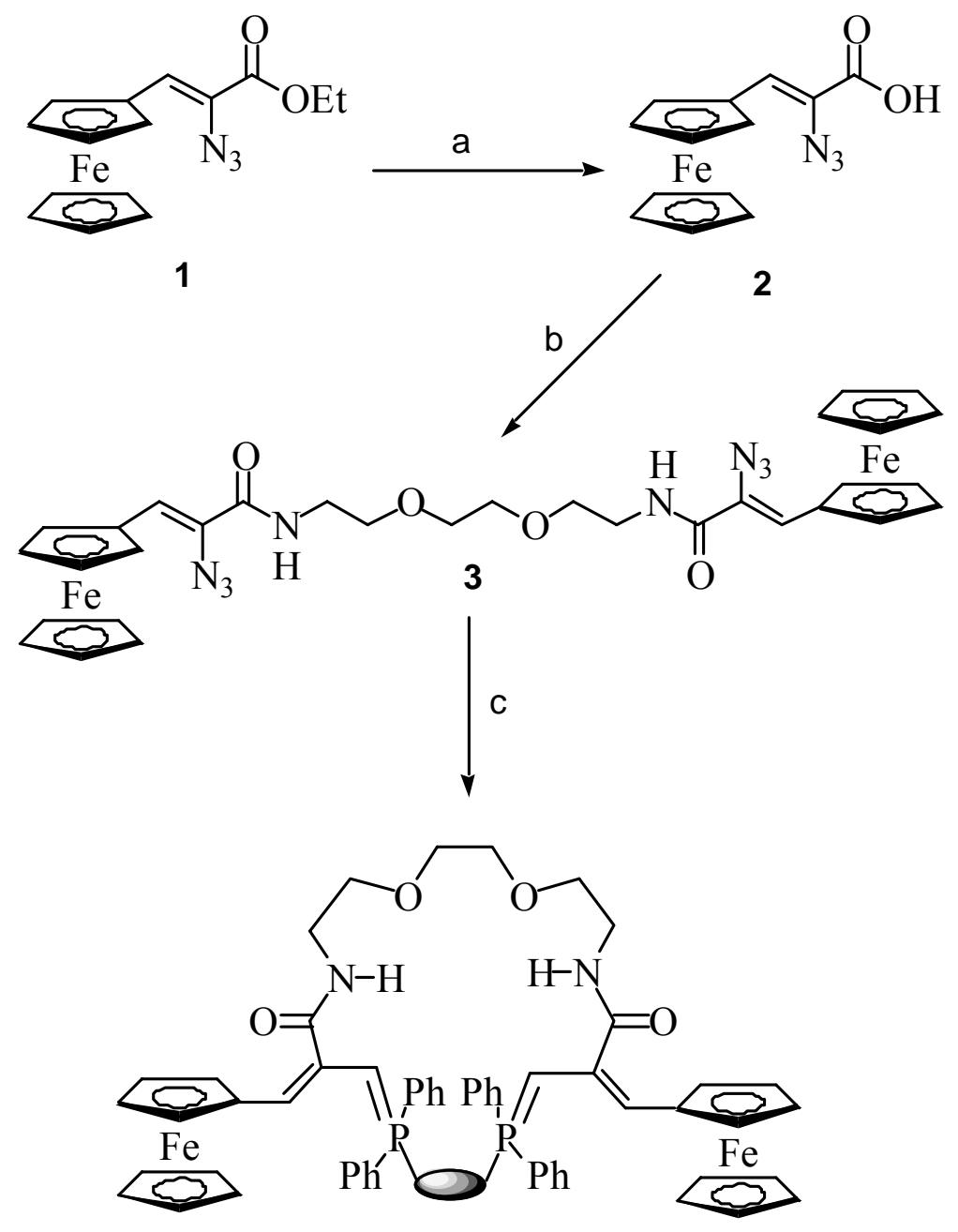

4

$\mathcal{Q}=-\left(\mathrm{CH}_{2}\right)_{\mathrm{n}^{-}}, 4 \mathrm{a}: \mathrm{n}=2 ; 4 \mathbf{b}: \mathrm{n}=3$

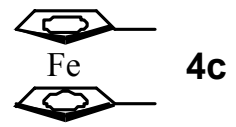

Scheme. (a) i) $\mathrm{KOH} / \mathrm{H}_{2} \mathrm{O}$, THF; ii) $\mathrm{HCl}(0.5 \mathrm{~N})$; (b) i) $\mathrm{ClCOCOCl} / \mathrm{CH}_{2} \mathrm{Cl}_{2}$ rt. $6 \mathrm{~h}$; ii) 2,2'(ethylenedioxy)bis(ethylamine)/Et ${ }_{3} \mathrm{~N}, \mathrm{THF}, \mathrm{rt}, 30 \mathrm{~min}$; (c) diphosphine/THF rt $24 \mathrm{~h}$.

The ${ }^{1} \mathrm{H}-\mathrm{NMR}$ analysis of compound $\mathbf{4 c}$ clearly shows two sets of well-separated signals for the two ferrocene subunits. The signals corresponding to the protons of the 1,1 'disubstituted ferrocene moiety appear at $\delta=4.24-4.28$ and $\delta=4.44-4.48$, while those corresponding to the protons of the two monosubstituted ferrocene moieties appear at $\delta=3.93$ and $\delta=4.40$, for the 
unsubstituted cyclopentadienyl ring, and $\delta=4.05$ for the monosubstituted ring. Similarly, the

${ }^{13} \mathrm{C}$-NMR spectrum shows the pattern for the two different types of ferrocene subunits present in the molecule and the FAB mass spectrum shows the expected $\left[\mathrm{M}^{+}+1\right]$ peaks at $\mathrm{m} / \mathrm{z}=1205$. The ${ }^{31} \mathrm{P}-\mathrm{NMR}$ spectrum shows one singlet at $\delta=2.18 \mathrm{ppm}$.

\section{Experimental Section}

General Procedures. All reactions were carried out under $\mathrm{N}_{2}$ and using solvents which were dried by routine procedures. Column chromatography was performed with the use of silica gel (60 A C.C. $70-200 \mathrm{~mm}$, sds) as the stationary phase.

All melting points were determined on a Kofler hot-plate melting point apparatus and are uncorrected. IR spectra were determined as Nujol emulsions on a Nicolet Impact 400 spectrophotometer. NMR spectra were recorded on a Bruker AC200 (200 MHz) or a Varian Unity $300(300 \mathrm{MHz})$. The FAB mass spectra were recorded on a Fisons AUTOSPEC $500 \mathrm{VG}$ spectrometer, using $m$-nitrobenzyl alcohol as a matrix. Microanalyses were performed on a Perkin-Elmer 240C instrument.

Preparation of 2-azido-3-ferrocenylacrylic acid (2). To a stirred solution of ethyl 2-azido-3ferrocenylacrylate $(0.20 \mathrm{~g}, 0.61 \mathrm{mmol})$ in THF $(15 \mathrm{ml})$ a solution of potassium hydroxide $(0.17 \mathrm{~g}, 2.5 \mathrm{mmol})$ in water $(6 \mathrm{ml})$ was added, and the mixture was stirred at room temperature overnight. The mixture was then acidified by adding a solution of hydrochloric acid $0.5 \mathrm{~N}$, and extracted with dichloromethane $(4 \times 20 \mathrm{ml})$. The organic extracts were combined and dried over $\mathrm{Na}_{2} \mathrm{SO}_{4}$. After filtration, the solvent was evaporated under reduced pressure and the crude product was scratched with $\mathrm{Et}_{2} \mathrm{O}$ to afford the product as a purple solid which was further purified by crystallization from dichloromethane $/ n$-hexane. Mp 117-119 ${ }^{\circ} \mathrm{C}$. Yield: 95\%. I.R (Nujol) $\mathrm{cm}^{-1}:$ 2930, 2125, 1681, 1616, 1425. ${ }^{1} \mathrm{H}-\mathrm{N} . \mathrm{M} . \mathrm{R}\left(\mathrm{CDCl}_{3}\right) \delta(\mathrm{ppm}): 4.18$ (s, 5H), 4.47 (s, 2H), 4.79 (s, 2H), $7.01(\mathrm{~s}, 1 \mathrm{H}), 8.42$ (bs, 1H). ${ }^{13} \mathrm{C}-\mathrm{N} . M . R\left(\mathrm{CDCl}_{3}\right) \delta(\mathrm{ppm}): 69.70(5 \mathrm{xCH}, \mathrm{Cp}-)$, 71.26 (2xCH, Cp-), 71.33 (2xCH, Cp-), 75.70 (Cq, Cp-), $121.15(\mathrm{Cq}), 130.98(\mathrm{CH}) 168.50(\mathrm{Cq}$, $\mathrm{C}=\mathrm{O}) . \mathrm{m} / \mathrm{z}$ : (rel intensity): $225\left(\mathrm{M}^{+}-\mathrm{CO}_{2}-\mathrm{N}_{2}, 100\right), 199$ (18), 121 (50), 78 (25), 56 (31). Anal. Calcd. for $\mathrm{C}_{13} \mathrm{H}_{11} \mathrm{FeN}_{3} \mathrm{O}_{2}$ : C, 52.56; H, 3.73; N, 14.14. Found: C, 52.80; H, 3.98; N, 13.91 .

Preparation of $N, N^{\prime}$-(3,6-dioxaoctanediyl)-bis(2-azido-3-ferrocenylacrylami-de) (3). To a stirred solution of 2-azido-3-ferrocenylacrylic acid $2(0.2 \mathrm{~g}, 0.67 \mathrm{mmol})$ in anhydrous dichloromethane $(6 \mathrm{ml})$ oxalyl chloride $(0.1 \mathrm{ml}, 1.2 \mathrm{mmol})$ was added, and the mixture was stirred at room temperature for $6 \mathrm{~h}$. The solvent was evaporated at reduced pressure and the crude product was washed with dry toluene $(3 \times 15 \mathrm{ml})$ and then dissolved in anhydrous THF $(10 \mathrm{ml})$. 2,2'-(Ethylenedioxy)bis(ethylamine) $(0.05 \mathrm{ml}, 0.34 \mathrm{mmol})$ and $\mathrm{dry}^{2} \mathrm{Et}_{3} \mathrm{~N}(0.09 \mathrm{ml}$, $0.67 \mathrm{mmol}$ ) were added and the mixture was stirred at room temperature and under nitrogen atmosphere for $30 \mathrm{~min}$. The reaction mixture was evaporated to dryness and the crude product was chromatographed on a silica gel column using $\mathrm{CH}_{2} \mathrm{Cl}_{2} / \mathrm{MeOH}(9 / 1)$ as eluent to give the 
product, which was crystallized from dicloromethane/diethyl ether. Mp $186-188^{\circ} \mathrm{C}$. Yield: $55 \%$. I.R (Nujol) $\mathrm{cm}^{-1}: 3343,2112,1651,1623,1538,1341,1285,1105 .{ }^{1}$ H-N.M.R $\left(\mathrm{CDCl}_{3}\right) \delta(\mathrm{ppm})$ : $3.56(\mathrm{t}, J=5.1 \mathrm{~Hz}, 4 \mathrm{H}), 3.66(\mathrm{t}, J=5.1 \mathrm{~Hz}, 4 \mathrm{H}), 3.68(\mathrm{~s}, 4 \mathrm{H}), 4.15(\mathrm{~s}, 10 \mathrm{H}), 4.37$ (t, $J=1.8 \mathrm{~Hz}$, $4 \mathrm{H}), 4.63(\mathrm{t}, J=1.8 \mathrm{~Hz}, 4 \mathrm{H}), 6.68(\mathrm{bt}, 2 \mathrm{H}), 6.70(\mathrm{~s}, 2 \mathrm{H}) .{ }^{13} \mathrm{C}-\mathrm{N} . M . R\left(\mathrm{CDCl}_{3}\right) \delta(\mathrm{ppm}): 39.66$ $\left(2 \mathrm{xCH}_{2}\right), 69.50(10 \mathrm{xCH}, \mathrm{Cp}), 69.65\left(2 \mathrm{xCH}_{2}\right), 70.23\left(2 \mathrm{xCH}_{2}\right), 70.30(8 \mathrm{xCH}, \mathrm{Cp}-), 76.41(2 \mathrm{xCq}$, $\mathrm{Cp}-), 123.63(2 \mathrm{xCH}), 126.78(2 \mathrm{xCq}), 163.37(2 \mathrm{xCq}, \mathrm{C}=\mathrm{O}) . \mathrm{FAB}^{+}, \mathrm{m} / \mathrm{z}$ : (rel intensity): 707 $\left(\mathrm{M}^{+}+1,12\right)$. Anal. Calcd. for $\mathrm{C}_{32} \mathrm{H}_{34} \mathrm{Fe}_{2} \mathrm{~N}_{8} \mathrm{O}_{4}: \mathrm{C}, 54.41 ; \mathrm{H}, 4.85 ; \mathrm{N}, 15.86$. Found: $\mathrm{C}, 54.20 ; \mathrm{H}$, $4.58 ; \mathrm{N}, 15.60$.

\section{General procedure for the preparation of macrocyclic bis-iminophosphoranes $\mathbf{4}$}

To a solution of bis-azide $3(0.2 \mathrm{~g}, 0.28 \mathrm{mmol})$ in dry THF $(15 \mathrm{ml})$ the appropriate diphosphine $(0.28 \mathrm{mmol})$ dissolved in dry THF $(3 \mathrm{ml})$ was added. The mixture was stirred at room temperature, under nitrogen, for $24 \mathrm{~h}$. The solvent was evaporated under reduced pressure to give the crude product which was purified by chromatography on a short silica gel column, eluting with $\mathrm{CH}_{2} \mathrm{Cl}_{2} / \mathrm{MeOH}(9 / 1)$. The products were crystallized from $\mathrm{CH}_{2} \mathrm{Cl}_{2} / \mathrm{Et}_{2} \mathrm{O}$.

4a. Mp 144-146 ${ }^{\circ} \mathrm{C}$. Yield: $82 \%$. I.R (Nujol) $\mathrm{cm}^{-1}: 3382,1652,1597,1440,1412,1122 .{ }^{1} \mathrm{H}-$ N.M.R $\left(\mathrm{CDCl}_{3}\right) \delta(\mathrm{ppm}): 2.57$ (bs, 4H), 3.23 (bd, $\left.J=4.8 \mathrm{~Hz}, 4 \mathrm{H}\right), 3.47$ (t, $\left.J=4.8 \mathrm{~Hz}, 4 \mathrm{H}\right), 3.55$ (s, $4 \mathrm{H}), 4.04(\mathrm{~s}, 4 \mathrm{H}), 4.06(\mathrm{~s}, 10 \mathrm{H}), 4.58(\mathrm{~s}, 4 \mathrm{H}), 6.22(\mathrm{~s}, 2 \mathrm{H}), 6.82(\mathrm{bt}, 2 \mathrm{H}), 7.30-7.57(\mathrm{~m}, 20 \mathrm{H})$. ${ }^{13}$ C-N.M.R $\left(\mathrm{CDCl}_{3}\right) \delta(\mathrm{ppm}): 21.57\left(\mathrm{t}, J=31.9 \mathrm{~Hz}, 2 \mathrm{xCH}_{2}\right), 39.44\left(2 \mathrm{xCH}_{2}\right), 67.72(4 \mathrm{xCH}, \mathrm{Cp}-)$, $68.95(10 x \mathrm{CH}, \mathrm{Cp}), 69.29(4 \mathrm{xCH}, \mathrm{Cp}-), 69.48\left(2 \mathrm{xCH}_{2}\right), 70.14\left(2 \mathrm{xCH}_{2}\right), 83.06(2 \mathrm{xCq}, \mathrm{Cp}-)$, $112.74(\mathrm{t}, J=7.4 \mathrm{~Hz}, 2 \mathrm{xCH}), 128.49\left(\mathrm{t},{ }^{3} J+{ }^{6} J=11.6 \mathrm{~Hz}, 8 \mathrm{xCH}_{\text {meta }}, \mathrm{Ph}-\mathrm{P}\right), 131.15\left(4 \mathrm{xC}_{\text {para }}, \mathrm{Ph}-\mathrm{P}\right)$, 131.37 ( $\left.\mathrm{t},{ }^{2} J+{ }^{5} J=8.8 \mathrm{~Hz}, 8 \mathrm{xC}_{\text {orto }}, \mathrm{Ph}-\mathrm{P}\right), 131.42-132.81$ (m, 4xC $\left.\mathrm{C}_{\text {ipso }}, \mathrm{Ph}-\mathrm{P}\right), 138.42$ (2xCq.), $169.44(2 \mathrm{xCq}, \mathrm{C}=\mathrm{O}) .{ }^{31}$ P-N.M.R. $\left(\mathrm{CDCl}_{3}\right) \delta(\mathrm{ppm}): 4.97 . \mathrm{FAB}^{+}, \mathrm{m} / \mathrm{z}$ : (rel intensity): 1049 $\left(\mathrm{M}^{+}+1,15\right), 1048\left(\mathrm{M}^{+}, 12\right)$. Anal. Calcd. for $\mathrm{C}_{58} \mathrm{H}_{58} \mathrm{Fe}_{2} \mathrm{~N}_{4} \mathrm{O}_{4} \mathrm{P}_{2}$ : C, 66.42; H, 5.47; N, 5.34. Found: C, 66.28; H, 5.26; N, 5.52 .

4b. Mp 123-125 ${ }^{\circ} \mathrm{C}$. Yield: yield: $80 \%$. I.R (Nujol) $\mathrm{cm}^{-1}: 3335,1668,1652,1592,1519,1438$, 1411, 1119, 1107. ${ }^{1}$ H-N.M.R $\left(\mathrm{CDCl}_{3}\right) \delta$ (ppm): 1.01 (bs, 2H), 2.46 (bs, 4H), 3.33 (bs, 4H), 3.50 (bs, 4H), 3.60 (s, 4H), 3.97-4.05 (m, 14H), 4.43 (s, 4H), 6.18 (s, 2H), 7.02 (bs, 2H), 7.31-7.57 (m, 20H). ${ }^{13}$ C-N.M.R $\left(\mathrm{CDCl}_{3}\right) \delta(\mathrm{ppm}): 15.13-15.74\left(\mathrm{~m}, \mathrm{CH}_{2}\right), 29.62\left(\mathrm{~m}, 2 \mathrm{xCH}_{2}\right), 39.41$ $\left(2 \mathrm{xCH}_{2}\right), 67.57$ (4xCH, Cp-), $68.86(10 \mathrm{xCH}, \mathrm{Cp}), 69.20(4 \mathrm{xCH}, \mathrm{Cp}-), 69.87\left(2 \mathrm{xCH}_{2}\right), 70.38$ $\left(2 \mathrm{XCH}_{2}\right), 83.35$ (2xCq, Cp-), 112.13 (d, J=16.7 Hz, 2xCH), 128.34 (d, J=11.9 Hz, 8xCH $\mathrm{CH}_{\text {meta }}$, PhP), 130.87 (4xCH $\mathrm{xara}_{\text {pa }}$ Ph-P), 131.19 (d, $J=9.2 \mathrm{~Hz}, 8 \mathrm{xCH}_{\text {orto }}, \mathrm{Ph}-\mathrm{P}$ ), 133.62 (upper peak of the, $4 \mathrm{xC}_{\text {ipso }}$ doublet, $\left.\mathrm{Ph}-\mathrm{P}\right), 138.46(2 \mathrm{xCq}), 169.50(2 \mathrm{xCq}, \mathrm{C}=\mathrm{O}) .{ }^{31} \mathrm{P}-\mathrm{N} . M . R .\left(\mathrm{CDCl}_{3}\right) \delta(\mathrm{ppm}): 1.17$. $\mathrm{FAB}^{+}, m / z$ : (rel intensity): $1065\left(\mathrm{M}^{+}+1,5\right), 1064\left(\mathrm{M}^{+}, 8\right)$. Anal. Calcd. for $\mathrm{C}_{59} \mathrm{H}_{60} \mathrm{Fe}_{2} \mathrm{~N}_{4} \mathrm{O}_{4} \mathrm{P}_{2}: \mathrm{C}$, 66.68; H, 5.69; N, 5.27. Found: C, 66.42; H, 5.90; N, 5.39.

4c. Mp 129-131 ${ }^{\circ} \mathrm{C}$. Yield: $85 \%$. I.R (Nujol) $\mathrm{cm}^{-1}: 3373,1652,1643,1593,1506,1411,1108 .{ }^{1} \mathrm{H}-$ N.M.R $\left(\mathrm{CDCl}_{3}\right) \delta(\mathrm{ppm}): 3.33(\mathrm{~m}, 4 \mathrm{H}), 3.58(\mathrm{t}, J=4.5 \mathrm{~Hz} ; 4 \mathrm{H}), 3.68$ (s, 4H), 3.93 (t, $J=1.8 \mathrm{~Hz}$, 4H), 4.05 (s, 10H), $4.26(\mathrm{~m}, 4 \mathrm{H}), 4.40$ (t, $J=1.8 \mathrm{~Hz}, 4 \mathrm{H}), 4.46$ (m, 4H), 6.53 (d, $J=1.8 \mathrm{~Hz}, 2 \mathrm{H})$, 7.28-7.55 (m, 20H) ${ }^{13}$ C-N.M.R $\left(\mathrm{CDCl}_{3}\right) \delta(\mathrm{ppm}): 39.80\left(2 \mathrm{xCH}_{2}\right), 67.64(4 \mathrm{xCH}, \mathrm{Cp}-)$, 68.88(10xCH, Cp), 69.31 (4xCH, Cp-), $69.89\left(2 \mathrm{xCH}_{2}\right), 70.26\left(2 \mathrm{xCH}_{2}\right), 72.85-73.45$ (m, 8xCH, 
Cp-), 75.33 (d, J=103.3 Hz, 2xCq, Cp-P), 83.24 (2xCq, Cp-), 113.87 (d, J=14.1 Hz, 2xCH

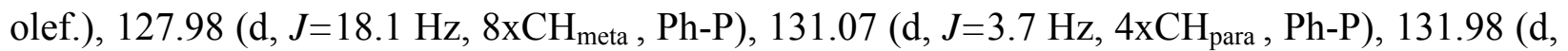
$\left.J=14.4 \mathrm{~Hz}, 8 \mathrm{xCH}_{\text {orto }}, \mathrm{Ph}-\mathrm{P}\right), 132.72$ (d, $\left.J=107.6 \mathrm{~Hz}, 4 \mathrm{xC}_{\text {ipso }}, \mathrm{Ph}-\mathrm{P}\right), 136.39$ (d, J=4.9 Hz, 2xCq), $169.58(2 \mathrm{xC}=\mathrm{O}) .{ }^{31}$ P-N.M.R. $\left(\mathrm{CDCl}_{3}\right) \delta(\mathrm{ppm}): 2.18 . \mathrm{FAB}^{+}, \mathrm{m} / \mathrm{z}:\left(\right.$ rel intensity): $1205\left(\mathrm{M}^{+}+1\right.$, 22), $1204\left(\mathrm{M}^{+}, 5\right)$. Anal. Calcd. for $\mathrm{C}_{66} \mathrm{H}_{62} \mathrm{Fe}_{3} \mathrm{~N}_{4} \mathrm{O}_{4} \mathrm{P}_{2}$ : C, 65.80; H, 5.19; N, 4.65. Found: $\mathrm{C}$, $66.06 ; \mathrm{H}, 5.03 ; \mathrm{N}, 4.88$.

\section{Acknowledgements}

Financial support was provided by the Dirección General de Investigación (MCYT, Spain) project BQU2001-0014 is warmly acknowledged with thanks.

\section{References}

1. For selected references, see: Hall, C.D, Ferrocenes Togni, A, Hayashi, T. Eds.; VCH: Weinheim, 1995; p 278.

2. (a) Constable, E. C. Angew. Chem. Int. Ed. Engl. 1991, 30, 407. (b) Beer, P. D. Adv. Inorg. Chem. 1992, 39, 79. (c) Medina, J. C.; Goodnow, T. T.; Rojas, M. T.; Atwood, J. L.; Lyn, B. C.; Kaifer, A. E.; Gokel, G. W. J. Am. Chem. Soc. 1992, 114, 10583. (d) Delavaux-Nicot, B.; Guari, Y.; Douziech, B.; Mathieu, R. J. Chem. Soc. Chem. Commun. 1995, 585. (e) Beer, P. D.; Graydon, A. R.; Sutton, L. R. Polyhedron 1996, 15, 2457. (f) Beer, P. D.; Szenes, F.; Balzani, V.; Sala, C. M.; Drew, M. G. B.; Dent, S. W.; Maestri, M. J. Am. Chem. Soc. 1997, 119, 11864. (g) Chesney, A.; Bryce, M. R.; Batsanov, A. S.; Howard, J. A. K.; Goldenberg, L.M. Chem. Commun. 1998, 677.

3. Abel, E. W., Mucklejohn, Phosphorus Sulfur 1981, 9, 235.

4. Dehnicke, K.; Strähle, J. Polyhedron 1989, 8, 707.

5. (a) Tárraga, A.; Molina, P.; López, J. L. Tetrahedron Lett. 2000, 41, 2479. (b) Tárraga, A.; Molina, P.; López, J. L. Organometallics (submitted).

6. (a) Avis, M. W.; Elsevier, C. J.; Veldeman, N.; Kooijman, H.; Spek, A. L. Inorganic Chem. 1996, 1518. (b) Avis, M. W.; Vrieze, A.; Ernsting, J. M.; Elsevier, C. J.; Veldeman, N.; Spek, A. L.; Katti, K. V.; Barnes, C. L. Organomet. Chem. 1996, 2376. (c) Avis, M. W.; van der Boom, C. J.; Elsevier, C. J.; Smeets, W. J. J.; Spek, A. L. J. Organomet. Chem. 1997, 527, 263. (d) Molina, P.; Arques, A.; García, A.; Ramirez de Arellano, M. C. Eur. J. Inorg. Chem. 1998, 1359.

7. (a) Staudinger, H.; Meyer, J. Helv. Chim. Acta 1919, 2, 635. (b) Golobov, Y. G.; Zhmurova, I. N.; Kasukhin, L. F. Tetrahedron, 1981, 37, 437.

8. (a) Tsvetkov, E. N.; Bovin, A. N.; Syundyukova, V. K. Uspekh. Khim. 1988, 57, 1353, Russ. Chem. Rev. 1988, 57, 776. (b) Izatt, R. M.; Pawlak, K.; Bradshaw, J. S.; Bruening, R. 
L. Chem. Rev. 1991, 91, 1721. (c) Caminade, A. M., Majoral, Chem. Rev. 1994, 94, 1183. (d) Molina, P.; Alajarín, M.; Arques. A.; Sánchez-Andrada, P.; Vidal, A.; Vinader, M. V. J. Organomet. Chem. 1997, 529, 121.

9. Mitjaville, J.; Caminade, A. M.; Mathieu, R.; Majoral, J. P. J. Am. Chem. Soc. 1994, 116, 5007.

10. (a) Hoppe, S.; Weichmann, H.; Jurkschat, K.; Schneider-Koglin, C.; Dräger, M. J. Organomet. Chem. 1995, 508, 209. (b) Goodwin, N. J.; Henderson, W.; Sarfo, J. K. J. Chem. Soc., Chem. Commun. 1996, 1551.

11. (a) Molina, P.; Pastor, A.; Vilaplana, M. J.; Ramírez de Arellano, M. C. Tetrahedron Lett. 1996, 37, 7829. (b) Molina, P.; Pastor, A.; Vilaplana, M. J.; Velasco, M. D.; Ramírez de Arellano, M. C. Organometallics 1997, 16, 5836. (c) Molina, P.; Tárraga, A.; Curiel, D.; Ramírez de Arellano, M. C. Tetrahedron 1999, 55, 1417

12. Skötsch C.; Breitmeier, E. Synthesis 1978, 680.

13. (a) Eguchi, S.; Yamashita, K.; Matsushita, Y.; Kakehi, A. J. Org. Chem. 1995, 60, 4006. (b) Okawa, T.; Eguchi, S. Tetrahedron Lett. 1996, 37, 81. (c) Velasco, M. D.; Molina, P.; Fresneda, P. M.; Sanz, M. A. Tetrahedron 2000, 56, 4079. 